applications and limitations. It gives a good intuitive account of what's involved in judging whether a given problem is computable, and an excellent explication of the Turing machine and the Church-Turing thesis (which says that Turing machines are capable of solving any effectively solvable algorithmic problem). It dips into parallel and quantum computing and cryptography and discusses the kinds of puzzles (the tiling problem, Towers of Hanoi, the monkey puzzle) that keep whole families fractiously occupied over Christmas. Whether it will induce an appropriate sense of humility in the computer industry, however, remains to be seen.

John Naughton is at the Open University, Milton Keynes MK7 6AA, UK. A Brief History of the Future, his book on the history and significance of the Internet, is published by Phoenix and Overlook Press in the United Kingdom and United States, respectively.

\section{Light on a distant subject}

\section{Star Clusters}

by Bruce W. Carney \& William E. Harris Springer: 2001. 417 pp. £44.50, \$69.95

\section{Carla Cacciari}

Not long after the Big Bang occurred, galaxies started to form. In this process stars also formed, as isolated individuals or bound into groups of up to several million stars. These spheroidal structures are called globular clusters and are interesting as fossil records of the early Universe. Younger clusters have also been found, showing that star formation can occur at later stages in the life of a galaxy. As all stars in a cluster are coeval and chemically alike, globular clusters are excellent laboratories for studying stellar evolution and for testing the models and physical assumptions underlying the theories. And last but not least, the properties of the cluster luminosity function are remarkably invariant from galaxy to galaxy, and so provide an excellent standard candle for determining distances.

Every year since 1971 the Swiss Society of Astrophysics and Astronomy has held a oneweek advanced course on an astronomical subject of general interest. Star Clusters is based on the lectures on globular star clusters given by Bruce Carney and William Harris at the 1998 course.

Bruce Carney's lectures are a good introduction to the evolution of stars in globular clusters. We meet the first basic concept in astrophysics: that stars that are born bigger evolve faster than less massive ones. Thus, we see stars in different evolutionary stages that tell us what it is like to be a teenage, adult or even a dead star in, say, a 14-billion-year-old

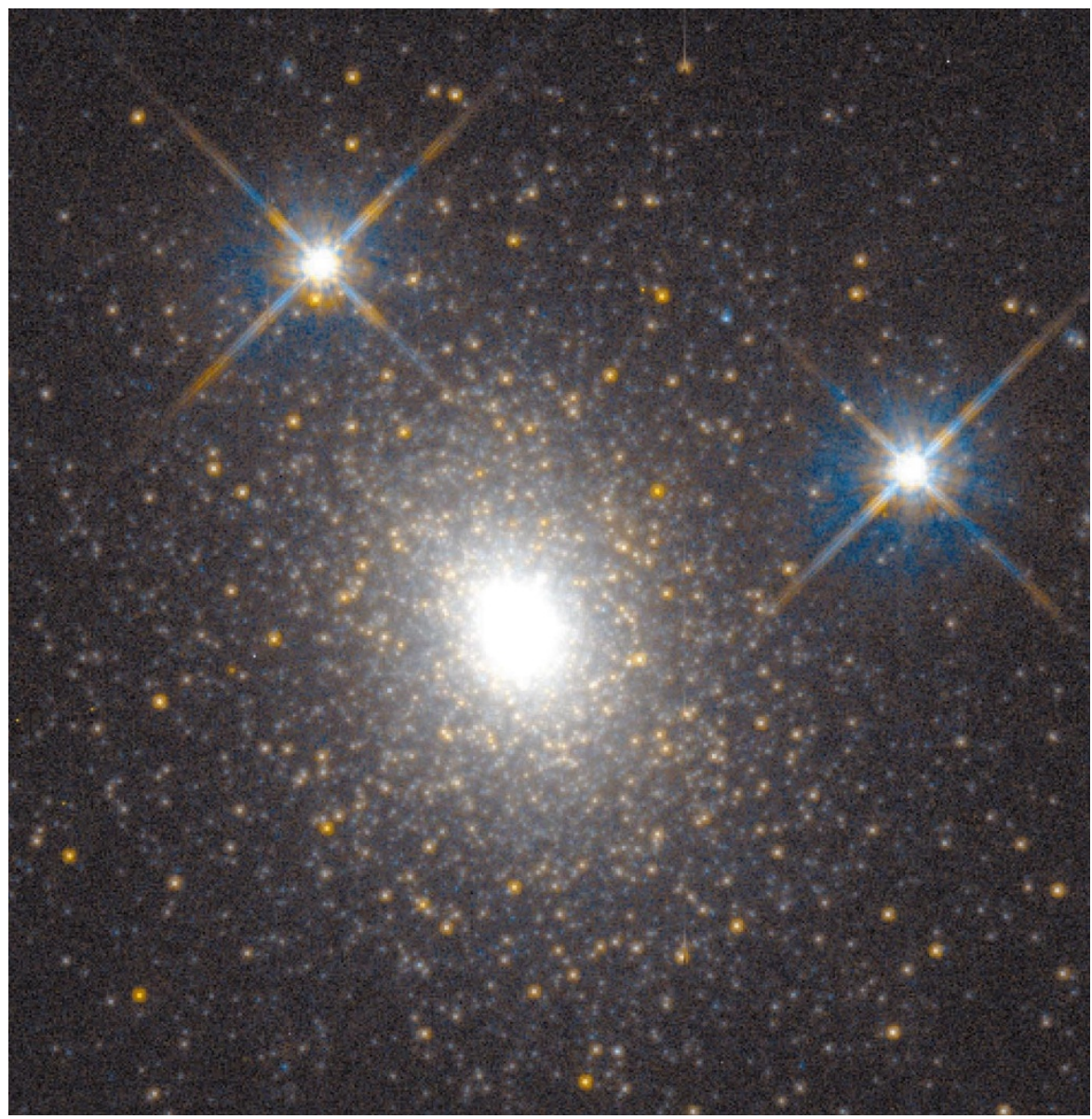

Globular clusters such as G1, in the Andromeda galaxy, tell us much about the early Universe.

globular cluster. Carney leads us skilfully through various technical details, unusual objects, and physical processes from basic nucleosynthesis to specific mixing phenomena in the stellar interiors. Along the way he discusses chemical and dynamical characteristics and their implications, which lead to the key item, the ages of the clusters. Relative ages among globular clusters, along with their chemical and dynamical properties, show us how the Galaxy formed, whereas the clusters' absolute ages give useful information about the age of the Universe.

Carney tells the story like a thriller. Some topics are threaded through the story and recur on different occasions, possibly with a slightly different look. Carney's views on other topics are occasionally stated with perhaps too much confidence. But even the most cautious investigators will feel that at some point they know who the murderer is, and so must read to the last page to learn whether they are right or wrong.

Harris considers globular clusters from another perspective: how they can be used to trace the processes of parent galaxy formation and evolution in our own Galaxy and also, as far as they have been identified, in external galaxies. His prose and method are clear and rigorous, and he leads us from one logical step to the next and on to the conclusion.

\section{New Journals}

This year, Nature's annual new journals review supplement will appear in the 18 October issue. Publishers and learned societies are invited to submit journals for review, as well as details of any eligible electronic journals, taking note of the following criteria:

- Journals must have first appeared during or after June 1999 and published at least 2001.

- Journals covering any aspect of science are eligible, although those dealing with clinical medicine and pure mathematics are excluded, as are newsletters and publications of abstracts.

- Frequency of publication must be at least three times a year.

- The main language must be English.

- Deadline for submission is 5 July.

For each eligible title, please send at least four different issues (the first, the most recent and any two others), together with full details of subscription rates, to: Isobel Flanagan, Nature, Porters South, Crinan Street, London N1 9XW, UK. Tel: +44 (0)20 7843 4542. Fax: +44 (0)20 7843 4596/7. e-mail: i.flanagan@nature.com four separate numbers by the end of June 
He starts by analysing the globular cluster system in the Milky Way, and its chemical, dynamic and kinematic properties. From these we can deduce the mass of the Galaxy and speculate on how it formed. We read again how the distance scale for globular clusters has been defined and critically evaluated using many different methods, and about the globular cluster luminosity function, which is a powerful tool for estimating extragalactic distances. Finally, we go beyond our own Galaxy to investigate the properties of the globular cluster systems in external galaxies - from Andromeda (the nearest) to those in the Virgo and Fornax clusters of galaxies. As the data have improved, so too has our understanding of galaxy formation, even though this brings with it an increasing complexity in the observational evidence and interpretative models. It is a great pleasure to read about this fascinating journey.

Astrophysics evolves fast, and some of the topics described as open questions or given tentative answers are now more firmly or somewhat differently answered; for example, what the relative luminosities of the stars RR Lyrae and Cepheid have revealed about the distance scale. Other such issues are the ages of globular clusters, or the fraction of evolved blue stars as a function of age.

The unavoidable repetitions between the two sets of lectures, far from being a drawback, offer the advantage of independent views of the same topic and how varied they can be.Together, the lectures present a great deal of information on globular clusters which is supported by precise technical detail, all the basic relevant formulae, statistical information on the available data and a wealth of references. The book is sure to become a basic reference source for scholars in this field for years to come.

Carla Cacciari is at the Osservatorio

Astronomico di Bologna, via Ranzani 1, 40127 Bologna, Italy.

Another recent book on stars and their evolution is Extreme Stars: At the Edge of Creation by James Kaler (Cambridge University Press: 2001. 236 pp. $£ 22.95, \$ 34.95)$, which looks at some of the extremes of stellar behaviour with the non-specialist reader in mind.

- Cataclysmic Variable Stars: How and Why They Vary by Coel Hellier (Springer-Praxis: 2001. 210

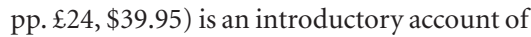
these binary stars for those with a background in astronomy or astrophysics.

- The Cambridge Dictionary of Space Technology by Mark Williamson (Cambridge University Press: 2001. 464 pp. $£ 27.95, \$ 39.95$ ) has 2,300 entries from A-4 ("the original engineering designation for the V-2") to Zvezda ("a module of the International Space Station") and "lists fundamental terms that will remain in common usage for the foreseeable future".

\section{Science in culture}

\section{The look of lightning}

Contrary to conventional belief, lightning is never zig-zag.

Martin Kemp

What does lightning look like? The way to find out now seems obvious. Simply take an 'instantaneous' photograph. This was exactly the solution proposed in the early 1880 s by the amateur photographer William Jennings, of Philadelphia. In a small notebook, now at the George Eastman House in Rochester, Jennings juxtaposed the varied, dendritic traces of actual lightning and thunderbolts with the stock portrayal by artists, which he called an "awkward zig-zag”. He proudly proclaimed that "for over fifteen years he [Jennings] made lightning photographs in various parts of the world; no two of which were alike, and none 'zig-zag'”.

The jagged shaft of conventional lightning still retains a strong popular hold, from Jupiter's handthrown thunderbolts, through warning signs of high voltage on pylons to logos of modern electricity companies. For showing the wrongness of the conventional zig-zag, Jennings was awarded the John Price Wetherill medal by the Franklin Institute - a particularly apposite award for a fellow Philadelphian of the great statesman and natural philosopher.

The truth was just as surely established by Eadweard Muybridge's famous serial photographs of galloping horses. The photographs, made for Governor Leland Stanford in California during the 1870s, had shown for ever the error of the artists' convention of a running horse with its four legs simultaneously reaching out in front and behind. The famous French painter of equestrian scenes, Ernest Meissonier, lamented that "all these years my eyes had deceived me. I find I have been wrong."

But are matters that simple? If we ask, "false for what?", the issue become less straightforward. The galloping horse's legs, like the flash of lightning, are too quick to be 'frozen' or seen in

discernible sequence by the human eye. If a representation is to evoke the way we actually see a rapidly moving object, it is the 'instantaneous' photograph that becomes false. It is rather like saying that $\mathrm{X}$-rays give the true picture of the human body rather than our customary vision of light on its exterior surface. If we can see any aspect of the galloping horse's legs, it is at the end of their travel, at the moment when all the legs are momentarily, if sequentially, stationary in front and behind.

Jennings's patterns of lightning are, if anything, even more problematic perceptually. Not only are the configurations infinitely varied, in a way that we now recognize as chaotic, but his photographic processes were actually rather slow compared to the lightning. What he captured was the course of the lightning over a definite time-span, through photography's equivalent of the persistence of vision. His process captured the linear trace that coursed from the heavens while the eye of his camera remained open and its retinal surface remained receptive.

We may readily recognize the forked menace of photographed lightning, just as the impressionist painters effectively exploited Muybridge's leg positions for a horse in rapid motion. However, the jagged zig-zag of convention still succeeds in working potently with the 'impression' produced when we stare at lightning using the perceptual apparatus with which we have been endowed. It also exudes a sense of graphic aggression that is highly expressive of lightning's destructive potential. We may know what lightning looks like, in an apparently objective sense, but how we actually see it is a very different matter. Martin Kemp is in the Department of the History of Art, University of Oxford, 59 George Street, Oxford OX1 2BE, UK.

The work of William Jennings can be seen in the exhibition Light!, which runs at the Carnegie Museum of Art in Pittsburgh until 29 July. 